\title{
Analysis of Ultrasonic Phased Array Signals Detected in CFRP
}

\author{
Zhan Xianglin, Cai Yujie, Liu Tao \\ College of Aeronautical Automation \\ Civil Aviation University of China \\ Tianjin, China \\ xlzhan@cauc.edu.cn
}

\begin{abstract}
Carbon Fiber Reinforced Plastic (CFRP) is an important material to make aircraft structure components. However, damage detection is very crucial to aircraft safety and the type of a detected defect is difficult to distinguish. Ultrasonic phased array (UPA) technology provides a possible solution. In this paper, characteristics of different defect signals and were studied in the frequency domain based on the wavelet packet analysis. Firstly, the principle of wavelet packet analysis is introduced. Secondly, a selection method of optimal wavelet on the basis of energy is proposed. The energy values of 41 wavelets are computed, and three kinds of wavelet basis are selected to extract the information entropy of the wavelet energy spectrum. Finally, a CFRP test block with four types of man-made defects is experimented. A-scan signals are collected by an UPA instrument. Information entropies are calculated and compared. From analysis, it can be seen that different types of defects can be differentiated by the corresponding entropy values. In addition, the entropy has high stability. In conclusion, information entropy can be used as an eigenvalue of UPA signals efficiently. This approach has laid the foundation for future research work.
\end{abstract}

Keywords- ultrasonic phased array ; nondestructive testing; carbon fiber reinforced plastic; signal processing; wavelet packet analysis

\section{INTRODUCTION}

Ultrasonic phased array (UPA) technology is a multibeam scanning and imaging technology. Every element of a transducer array is controlled by the electronic system. Ultrasonic signals are transmitted or received according to a certain delaying time rule. The rule is critical because it controls the focus position and the deflection direction of the transmitted beams in the tested object, which finally determines the results of defect detection [1-3]. Therefore, the UPA technology makes test results more intuitive and the quantitative defect analysis more efficient. However, the type of a detected defect is difficult to distinguish from the current ultrasonic images. To solve this problem, a new method is proposed to analyze the UPA signals detected from the CFRP. In this method, characteristics of different types of detection signals are studied, and feature vectors are extracted and analyzed. Existing feature analysis methods are focused on time-domain analysis, timefrequency domain analysis and frequency domain analysis. On the other hand, the spectral analysis techniques, timefrequency analysis, wavelet transform and adaptive filters are widely used in feature extraction field. In applications, a key research interest is to use wavelet transform to realize feature extraction [4-7].

In this paper, a CFRP test block has been made, in which four types of artificial defects have been processed too. The defects are made of monolayer Teflon film, the bilayer Teflon film, glass ball and a foil. After nondestructive testing is carried out, the time and frequency characteristics of the detection signals are analyzed by the wavelet packet analysis. Information entropies are calculated and can be seen as the information measures of different types of UPA signal. The proposed feature extraction method based on information entropy is effective in qualitative defect analysis on CFRP by UPA testing

\section{WAVELET PACKET ANALySis OF A DETECTION SIGNAL}

Because an ultrasound detection signal is a nonstationary signal, signal features extracted through time domain analysis or frequency domain analysis cannot fully reflect the signal characters. However, the wavelet packet analysis method is an extension of the wavelet analysis. It is a series of wavelet decomposition, which offers a richer signal analysis and is suitable to be used for signal reconstruction [8]. A signal is decomposed into a lowfrequency domain and a high-frequency domain by a wavelet packet transform. After decomposed several times, the signal is divided into multiple levels. Both the lowfrequency and the high-frequency parts of the signal are decomposed at the same time. Therefore, the signal can not only be observed in a more detailed way by this multiresolution analysis, but be matched by selecting the corresponding frequency bands according to its characteristics. In this way, the frequency resolution can be increased and the features of the testing ultrasonic signal can be extracted more clearly.

Wavelet packet function is defined as follows [9]. Given orthogonal scaling function $\varphi(t)$ and wavelet function $\psi(t)$, their two-scale relationship is:

$$
\phi(t)=\sqrt{2} \sum_{k} h_{0 k} \phi(2 t-k) .
$$




$$
\psi(t)=\sqrt{2} \sum_{k} h_{1 k} \phi(2 t-k)
$$

$h_{0 k}$ and $h_{l k}$ are filter coefficients of multi-resolution analysis.

Based on (1) and (2), a recurrence relation can be obtained:

$$
\begin{aligned}
w_{2 n}(t) & =\sqrt{2} \sum_{k \in z} h_{0 k} w_{n}(2 t-k) . \\
w_{2 n+1}(t) & =\sqrt{2} \sum_{k \in z} h_{1 k} w_{n}(2 t-k) .
\end{aligned}
$$

In (3), when the value of $n$ is zero, $w_{0}(t)$ is $\varphi(t)$. In (4), $w_{1}(t)$ is $\psi(t)$ as $n$ is zero. So, the set of functions $\left\{w_{\mathrm{n}}(t)\right\}_{\mathrm{n} \in \mathrm{Z}}$ is the wavelet packet in fact, which is made up of the scaling function $w_{0}(t)$ and the mother wavelet $w_{1}(t)$.

Therefore, the wavelet packet decomposition algorithm can be obtained as follows:

$$
\begin{gathered}
d_{l}^{j, 2 n}=\frac{1}{\sqrt{2}} \sum_{k} h_{k-2 l} d_{k}^{j+1, n} . \\
d_{l}^{j, 2 n+1}=\frac{1}{\sqrt{2}} \sum_{k} g_{k-2 l} d_{k}^{j+1, n} .
\end{gathered}
$$

In addition, the reconstruction algorithm based on wavelet packet is:

$$
d_{l}^{j, 2 n+1}=\frac{1}{\sqrt{2}} \sum_{k}\left\{h_{l-2 k} d_{k}^{j, 2 n} g_{l-2 k} d_{k}^{j, 2 n+1}\right\}
$$

It can be seen that as the value of $j$ increases, the spatial resolution of corresponding orthogonal wavelet function is higher, but the frequency resolution gets lower, which is a major drawback of orthogonal wavelet bases. However, by the wavelet packet decomposition, the frequency band will be further divided as $j$ increases. Hence, it can overcome the deficiency of orthogonal wavelet transform. Moreover, a higher frequency resolution and more frequency-domain information can be obtained, which is better for extracting feature information.

\section{FEATURE INFORMATION EXTRACTION BASED ON WAVELET ENERGY ENTROPY}

An ultrasonic echo collected by the UPA system is an A-scan image. In Fig .1, there are four A-scan images of four types of defects collected by eight array elements shown. From Fig .1 we can see that echo shapes of the glass ball and the foil are very similar and are not easy to be distinguished by the time-domain graph. Meanwhile, the echo shapes of the monolayer Teflon film and the bilayer Teflon film are very similar too. From this point of view, it is very important to extract distinguishable feature information.

In this paper, the wavelet energy entropy is computed as a feature extraction vector. According to the above analysis, four kinds of echo signals are divided into two groups: the glass ball and the foil are a group while the monolayer Teflon film and the bilayer Teflon film are the other. Through analysis of these two groups, feature information of defects in CFRP will be got.

\section{A. Wavelet packet selection based on energy difference principle}

In order to obtain the stable entropy information of the detected, an optimum wavelet packet basis needs to be determined. Therefore, the detection signal can be decomposed efficiently. Then the information entropy can be computed based on the decomposed time-frequency signal. Because decomposition results have a decisive effect on stability of information entropy, obtaining an optimum wavelet packet basis is very important. Furthermore, entropy reflects the signal energy distribution characteristics in different transform space and the energy distribution difference and separability of the sequence as well [10]. Accordingly, the principle of energy difference for the detection signal is used to select wavelet basis, that is, the optimum wavelet basis makes the energy differences between the various sub-space maximum while the energy in the subspace minimum.

Accordingly, four types of defect signals are decomposed by the wavelet packet analysis. Energy differences are calculated between different subspaces, which are the standard of selecting wavelet packet basis. The specific process is as follows. Suppose there is a signal sequence $f(t)$. Its generalized transform space is divided into $I$ feature subspaces, then the difference of the signal energy between the $i^{\text {th }}$ and the $j^{\text {th }}$ subspace can be expressed as:

$$
\Delta_{i, j}=\left|E_{i}-E_{j}\right| / \max \left(\left|E_{i}\right|,\left|E_{j}\right|\right), i \neq j .
$$

In (8), $E_{i}$ and $E_{j}$ represent the signal energy sum of the $i^{\text {th }}$ and the $j^{\text {th }}$ subspace respectively, which can be defined as:

$$
\mathrm{E}_{\mathrm{i}}=\int|\mathrm{f}(\mathrm{t})|^{2} \mathrm{dt}=\sum_{j=1}^{n}\left|X_{i j}\right|^{2} .
$$

$X_{i j}$ is the amplitude of each point in the $i^{\text {th }}$ space.

According to the rule of maximum energy difference between various subspaces, a fitness function $F_{a}$ can be constructed which can indicate the difference degree of signals' energy between various feature spaces.

$$
\mathrm{F}_{\mathrm{a}}=\sqrt{\frac{1}{2} \sum_{j=1}^{l} \sum_{i=1}^{l}\left(\Delta_{i j}\right)^{2}} .
$$

Similarly, a fitness function $F_{b}$ can be constructed, which indicates the difference degree of signals' energy inside the feature space. 

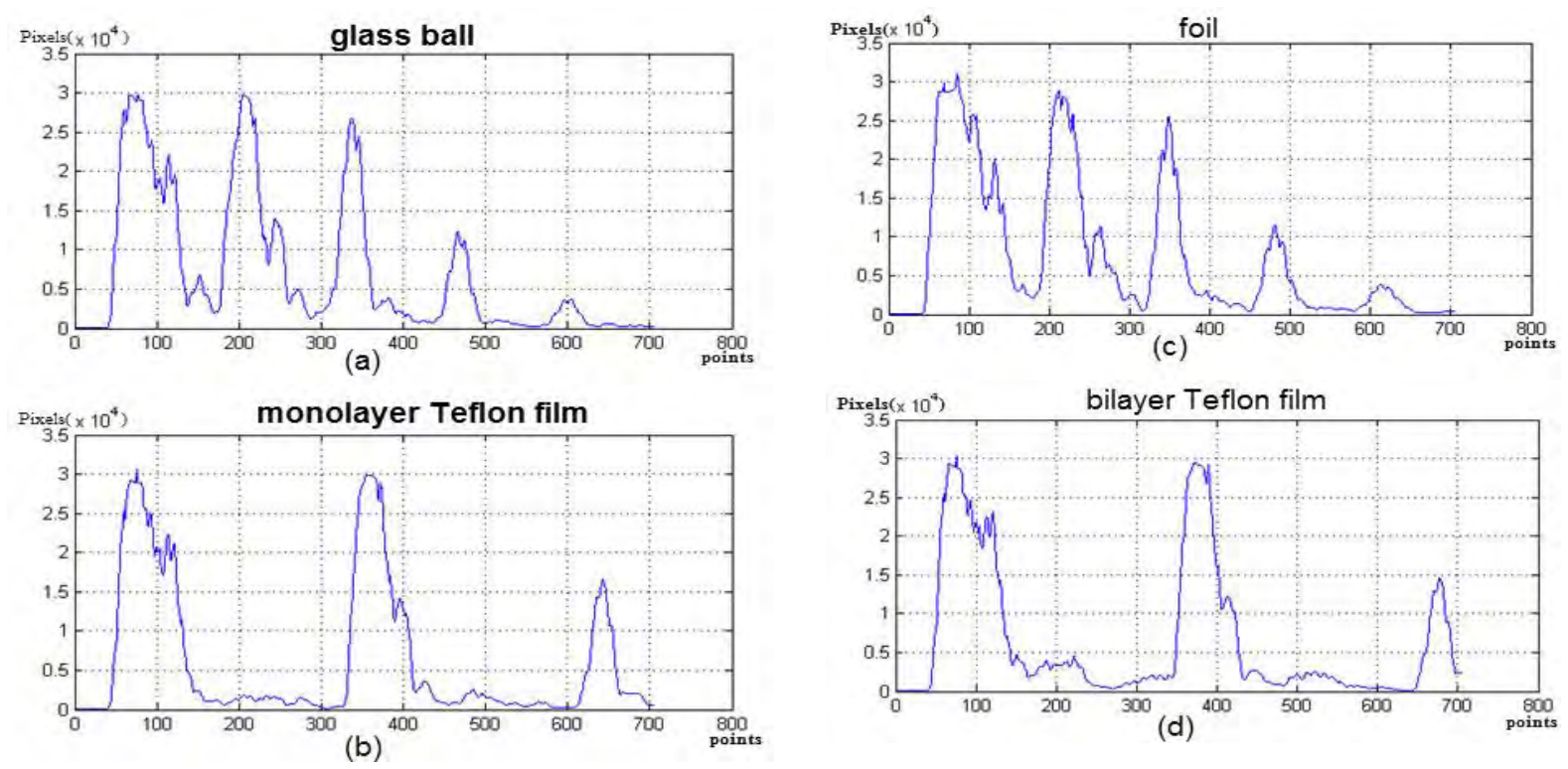

Figure 1. A-scan signals of four types of defects in CFRP testing block.

$$
\mathrm{F}_{\mathrm{b}}=\sqrt{\sum_{i=1}^{l} \Delta_{i, i}^{2}}
$$

where $\Delta_{i, j}=\sigma_{i}^{2} /\left(\sigma_{i}^{2}+\mu_{i}^{2}\right), \sigma_{i}$ is the standard deviation of the signal in each subspace and $\mu_{i}$ is the mean value of the signal in each subspace. Based on the values of $F_{a}$ and $F_{b}$, the wavelet packet basis can be selected, which means the value of $F_{a}$ should be as large as possible whereas the value of $F_{b}$ should be as small as possible.

However, it is easy to confuse when $F_{a}$ and $F_{b}$ are considered at the same time. Hence, a comprehensive fitness function $F$ is constructed, whose value range is $(0$, 1 ). When $F_{a}$ is the maximum and $F_{b}$ is the minimum, $F$ is close to one. So it can be referred that if the value of $F$ is closer to one, the fitness is better, which means the optimum wavelet packet basis is selected. The definition of $F$ is shown in (12).

$$
F=\left\{\begin{array}{l}
\frac{F_{a}-F_{b}}{F_{a}}, \max \left(F_{a}, F_{b}\right)=F_{a} \\
1-\frac{\left|F_{a}-F_{b}\right|}{F_{b}}, \max \left(F_{a}, F_{b}\right)=F_{b}
\end{array} .\right.
$$

\section{B. Feature extraction of information entropy}

Wavelet energy entropy is selected as the feature and is extracted after the signal is processed by the wavelet packet analysis. Because an ultrasound testing signal is a finite energy signal, it satisfies the energy conservation law after the wavelet packet transform, which can be expressed as (13).

$$
\int_{-\infty}^{+\infty}|f(\mathrm{t})|^{2} d t=K \int_{0}^{+\infty} a^{-2} E(\mathrm{a}) d a
$$

where $E(a)$ is the energy value of function $f(t)$ at scale $a$, which is called as the wavelet energy. And for the discrete signals, it is known that:

$$
E(\mathrm{a})=\int_{-\infty}^{+\infty}\left|W_{f}(\mathrm{a}, \mathrm{b})\right|^{2} d b=\sum_{b=1}^{n}\left|W_{f}(\mathrm{a}, \mathrm{b})\right|^{2}
$$

Therefore, the signal $\mathrm{f}(\mathrm{t})$ decomposed at $\mathrm{n}$ scale, its wavelet energy spectrum can be defined as $E=\left[E_{1}\right.$, $\left.E_{2}, \ldots, E_{\mathrm{n}}\right]$, which makes an energy division of the signal in the time-frequency domain. And the corresponding wavelet energy entropy $H_{E}$ can be defined as (15).

$$
H_{E}=-\sum_{i=1}^{n} p_{i} \ln p_{i}, p_{i}=E_{i} / \sum_{i=1}^{n} E_{i} .
$$

where $p_{i}$ represents the proportionate of the energy at scale $i$ that occupies the ratio of the entire energy. $H_{E}$ is considered as the eigenvalues of different types of UPA detected signals.

\section{EXPERIMENTS}

A UPA system is used to test a CFRP test block, in which there are four types of manual defects, i.e., monolayer Teflon film, the bilayer Teflon film, glass ball and a foil. From all the collected images, an image was randomly selected from each type of defect, as shown in Fig .1. Four signals are divided into two groups as mentioned above.

Then, the selected four signals are processed by the proposed method to obtain the feature $H_{E}$. First, an optimum wavelet packet basis should be selected after the UPA signals are decomposed by the wavelet packet algorithm. Then, the comprehensive fitness $F$ is computed. The values of $F$ of the glass ball and the foil are shown in Fig .2 and those of the monolayer Teflon film and the bilayer Teflon film are shown in Fig .3. 


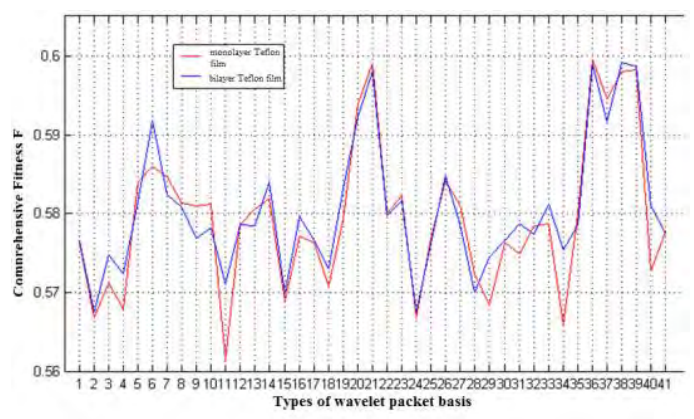

Figure 2. Monolayer and bilayer Teflon film's F

From Fig .2, we can see that when the $36^{\text {th }}$ wavelet basis is chosen, the corresponding comprehensive fitness $F$ is the largest. So bior3.1 wavelet basis is chosen. As shown in Fig .3, when the $21^{\text {th }}$ and $39^{\text {th }}$ wavelet bases were chosen, the values of $F$ are both large. So rbio3.9 and bior3.9 are both chosen.Then take the four layer wavelet packet decomposition, the signals' entropy values are obtained and shown in Table I.

TABLE I. VALUES OF INFORMATION ENTROPY

\begin{tabular}{|l|c|c|}
\hline \multicolumn{1}{|c|}{ Defect Type } & Wavelet Packet Basis & Entropy $\boldsymbol{H}_{\boldsymbol{E}}$ \\
\hline Monolayer Teflon film & bior3.1 & 0.054306 \\
\hline Bilayer Teflon film & bior3.1 & 0.054825 \\
\hline Foil & rbio3.9 & 0.134079 \\
\hline Glass ball & rbio3.9 & 0.115444 \\
\hline Foil & bior3.9 & 0.081810 \\
\hline Glass ball & bior3.9 & 0.086497 \\
\hline
\end{tabular}

From Table I, we can see that different types of manual defects in the CFRP testing block have different entropy values, and the entropy has high stability. The entropy values of monolayer Teflon film and bilayer Teflon film are normally less than those of glass ball and foil. This makes it easy to discriminate the former from the latter. In addition, even in one group, it can tell from what kind of signal is based on the value of $H_{E}$. For example, the entropy value of monolayer Teflon film is less than that of bilayer Teflon film. Therefore, entropy is an effective feature

\section{CONCLUSION}

When the CFRP composite buried with different kind of defects inspected by an UPA system, it is difficult to distinguish the defects only according to the time domain waveform of the obtained A-scan signal. To solve this problem, a method based on wavelet packet transform combined with entropy analysis is proposed in this paper. The wavelet packet transform carries out the time-frequency analysis of A-scan signals, and then the spectrum information entropy are extracted from time - frequency analysis results. As values of entropy indicate the type of defects, entropy is seen as the feature of defect signals. In addition, information entropy values are different if the signal is decomposed by different wavelet packet basis. Therefore, 41 kinds of wavelet bases have been evaluated. Accordingly, bior3.1

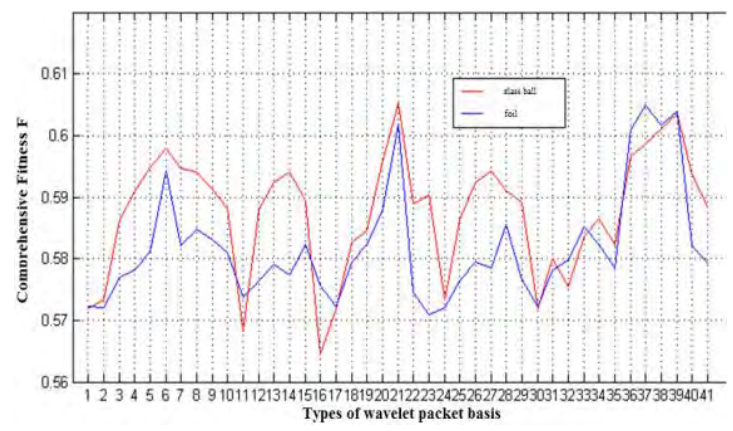

Figure 3. Glass ball and foil's F

wavelet basis is chosen to distinguish from the glass ball and the foil. At the same time, rbio3.9 and bior3.9 are chosen to discriminate the monolayer Teflon film from the bilayer Teflon film. Finally, by calculating the entropy, it is easy to find that different types of defects can be distinguished by their entropy values. This approach that takes information Entropy as an eigenvalue of A-scan signal has laid a foundation for subsequent research of automatic defect recognition.

\section{ACKNOWLEDGMENT}

Zhan Xianglin would like to thank National Natural Science Foundation of China (Grant NO. 61102097 and 61405246), Tianjin Science and Technology Support Program, China (Grant NO. 11ZCGHHZ00700) and Central Universities Fundamental Research Grant (SY1418 and 3122014D023) to support.

\section{REFERENCES}

[1] R. Bulgrin, S. Goldbach, and R. Bulgrin, "Visualization of CFRP structures based on acoustic techniques for NDT," Proc. 2nd International Symposium on NDT in Aerospace, Nov., 2010.

[2] Smith R A, and Nelson L J, "Automated analysis and advanced defect characterization from ultrasonic scans of composites," Insight, vol. 51, Feb. 2009, pp. 82-87.

[3] S. Iyer, S.K. Sinha, and B.R. Tittmann, "Ultrasonic signal processing methods for detection of defects in concrete pipes," Automation in Construction, vol. 22, 2012, pp. 135-148.

[4] Zhang Ping, Shi Ke-ren, and Geng Rong-sheng, "Application of Wavelet Transform in Acoustic Emission Testing," NDT, vol. 24, Oct. 2002, pp. 436-442.

[5] Zhao Xia, and Wang Zhao-ba, "Research on optimization of digital phased array ultrasonic testing system independent channel number," Electronic Testing, vol. 9, 2014, pp.34-36.

[6] Y Zhang, and L Yang, "Study on feature extraction and classification of ultrasonic flaw signals," WSEAS Transactions on Mathematics, vol. 9, 2010, pp. 529-538.

[7] Gabriela Pèrez, Aura Conci, Ana Belén Moreno, Juan Antonio, and Hernandez-Tamames, "Rician noise attenuation in the wavelet packet transformed domain for brain MRI," Integrated Computer-Aided Engineering, vol. 21, Apr. 2014, pp. 163-175.

[8] Dong Jian-liang, Feng Hong, and Ma Chen-guang, "Analysis of signal denoising for rayleigh wave based on wavelet transform," Electronic Testing, vol. 10, 2013, pp.100-103.

[9] B. Donald, Percival and Andrew T. Walden, "Wavelet methods for tome series analysis," Cambridge: Cambridge University Press, 2000.

[10] Xie Ping, Liu Bin, and Lin Hong-bin, "Study on multiresolution singular-spectrum entropy and its usage in vibration signal monitoring," Journal of Translation Technology, vol. 17, Apr. 2004, pp. 548-550. 